\title{
MIR429 Pre-miRNA
}

National Cancer Institute

\section{Source}

National Cancer Institute. MIR429 Pre-miRNA. NCI Thesaurus. Code C82830.

MIR429 is an oligoribonucleotide that is encoded by the human MIR429 gene and has a role in the regulation of gene expression. 